\title{
Defensive Behaviour in Shelter Dogs
}

\author{
Timea Andrea KOCIS ${ }^{1}$, Ioan ȚIBRU ${ }^{1}$ \\ ${ }^{1}$ Banat's University of Agricultural Science and Veterinary Medicine Timisoara "King Michael of \\ Romania", Faculty of Veterinary Medicine, 300645, Calea Aradului, no 119, Timisoara, Romania \\ Corresponding author: dea16_tim@yahoo.com
}

Bulletin UASVM Veterinary Medicine 72(1) / 2015,

Print ISSN 1843-5270; Electronic ISSN 1843-5378

DOI:10.15835/buasvmcn-vm: 10475

\begin{abstract}
Dogs in shelters are exposed to a new environment, and to permanent stress factors, they interact with foreign dogs and persons. These factors lead to behaviour disorders, including the occurrence of a defensive aggressive behaviour. This type of aggression will be observed in individuals which are fearful, insecure in certain circumstances; that is why, as a means of protection, they will use aggression. Theses tests aim to create an inventory and an identification of behaviours specific to aggressive defensive dogs, as a result of their being accommodated in shelters. This study includes a number of 20 dogs, selected from a number of 200 dogs living permanently in a shelter in the city of Timisoara. Experiments were registered with a video camera; the images were processed and interpreted. Defensive behaviour evaluation was based on a test comprising several criteria. During the first test, the evaluator tried to establish a visual contact with the dog. The second defensive behaviour evaluation criteria consisted in the attempt to approach the dog and to communicate verbally with it. During the third test, the physical contact between evaluator and dog was sought, through stroking.

During the first stage, a number of 10 dogs immediately established eye contact with the evaluator, 5 avoided eye contact, 2 dogs accepted visual contact but refused physical contact, 3 dogs stepping away from the evaluator. During the second stage, 12 dogs responded favourably, 5 dogs allowed the touch, 3 dogs not allowing the touch. During the last stage, 11 dogs have accepted stroking, showing no signs of aggressiveness stemming from fear, 4 accepted the touch, 5 refused this type of interaction.

Due to the environmental conditions in shelters, these dogs may respond in an unfavourable way, displaying defensive aggression. Through body language knowledge and defensive dog behaviour identification, one can avoid unpleasant situations when these dogs become aggressive.
\end{abstract}

Keywords: aggression, defensive, dog.

\section{INTRODUCTION}

Shelter dogs are exposed to a new, changing environment, always in the presence of stress factors, interacting with foreign dogs and persons. These factors lead to the appearance of behaviour disorders, including aggressive behaviour due to anxiety (Christensen et al., 2007).

This kind of aggression will be observed with individuals that are anxious, unsure in certain circumstances, that is why they will use aggressiveness as a defence mechanism. If the warning signs are not understood by the shelter staff or other persons, the dog will bite with no apparent cause. Aggressiveness is a coping mechanism, its purpose being the survival of the individual. Aggressiveness states in dogs are closely linked to the level, limit and capacity to adapt to environmental factors, and its nervous excitement degree (Bollen and Horowitz, 2008). The test aims to achieve an inventory, as well as recognition of specific anxious, defensive dog behaviour, which are predisposed to aggressive manifestation as a result of accommodating them in shelters. 


\section{MATERIALS AND METHODS}

This study included a number of 20 dogs ( 10 male and 10 female), selected from a number of dogs permanently kept in a shelter of the city of Timisoara. The following dog selection criteria were established: the duration of being kept in a shelter (at least 6 months), age (1-7 years old), gender, size, health state (sick animals were excluded) and aggressiveness. As a starting point we used information on the dog behaviour history: their reaction to the staff, to foreign persons (visitors), the reaction to human closeness while feeding (accept/refuse), accepting physical contact with people or not. If a dog manifested aggressive behaviour (growling, chattering, biting) in one of these situations, it was considered that he could not be included in the experiment, being deemed dangerous for the examiner for the time being (Bollen and Horowitz, 2008).

This is the reason why 20 dogs were selected from the total number, including not only wild dogs from the streets, but also dogs abandoned by their masters for various reasons. The owners who brought their dogs to the shelter offered information on the dogs' behaviour history, according to the studies (Bollen and Horowitz, 2008).

The examination mode, respectively the dogs' behaviour, was video-recorded, the images being processed and interpreted subsequently. The evaluation team comprised two members, an evaluator and a person doing the recording.

Dogs were accommodated in boxes, sheltering no more than four dogs, in accordance with the legally required space and wellbeing conditions.

The preliminary evaluation took place in the sheltering boxes, each dog being observed for 10 minutes, before being brought to the evaluation room. The subjects were evaluated individually then brought by the shelter staff from their boxes to the evaluation room, with a $5 \times 4$ size. In the end, a behavioural inventory (ethogram) was established, comparing posts with data from specialty literature (De Meester et al., 2008).

After they have been brought to the examination room, each dog has been given 5 minutes to get used to the environment. During this time, the room was kept quiet, no discussions took place and there were no sudden moves (Christensen et al., 2007).
Because only relatively calm dogs have been selected for the study, one could observe only defensive aggressiveness after the evaluation. In order to establish whether the studied dogs presented aggressive behaviour, the team monitored their answer to:

- during the first stage, the evaluator tried to establish eye contact with the dog. Thus, dogs that establish eye contact in less than 5 seconds, and allowed to be touched were marked 2 , and dogs that did not allow eye contact but allowed physical contact were marked 1 . The ones allowing eye contact but not the physical one were marked minus 1 , and the ones that did not allow either eye or physical contact received a minus 2.

- during the second stage, defensive aggressiveness was evaluated by evaluating the degree of acceptance when approaching the dog simultaneously with voiced communication. That is why dogs that allowed the touch while being spoken to received a 3 , dogs that did not allow the touch while being spoken to received a 2, and the ones that allowed the touch but without being spoken to received a 1 .

- during the third stage, acceptance of prolonged physical contact between the evaluator and the dog, through stroking. Dogs which accepted the stroke and established a positive contact with the evaluator, received a 3 , those who accepted the stroke but moderately received a 2 , and the ones that refused and showed signs of discomfort received a 1.

Final interpretation was accomplished based on the evaluated body language and the videos (Bollen and Horowitz, 2008).

\section{RESULTS AND DISSCUSIONS}

During the first stage, a number of 10 dogs, marked with a 2 , immediately established eye contact with the evaluator, in less than 5 seconds; these displayed a positive attitude: perked up ears, alert look, directed towards the evaluator.

- 5 avoided eye contact by turning their head, thus receiving a 1 , with the characteristic half moon look, but accepted physical contact, even though showing moderation manifested by muscular tension.

- 2 dogs accepted visual contact, but for a brief time, and were marked with a minus 1 , but refused physical contact, dodging the evaluator's hand. 
- 3 dogs stepping away from the evaluator received a minus 2 , showing a fearful, anxious attitude by withdrawing to a corner of the room.

Analyzing the data obtained after the firststage, we can say that 10 dogs responded favourably, by accepting eye contact as well as physical one, manifesting a positive, relaxed attitude: alert look, perked up ears, showing interest, vigorous tail wagging. The two dogs which refused the physical contact (the evaluator's touch) reacted that way because of lack of trust. The dogs refusing any kind of contact with the evaluator were of the anxious kind, which prefer to withdraw, due to their distrust of people (Fig.1).

During the second stage, 12 dogs responded favourably, receiving a 3 . They immediately accepted the interaction, answering the evaluator's call, immediately walking to him and enjoying the strokes. From a behavioural point of view, the dogs were relaxed, vigorously wagging their tail, some even manifesting playing behaviour. 5 dogs allowed the touch without being spoken to, receiving a 2; 3 dogs not allowing the touch while being spoken to received a 1 . These withdrew the moment the evaluator reached out with his hand.

As a result of the second stage, we can say that 12 dogs responding to the call and not manifesting any sign of aggression or anxiety may be offered up for adoption. The 3 dogs which did not allow the touch displaying signs of discomfort, such as lip licking, characteristic half moon look, hair raised in the croup region (Fig. 2).

During the last stage, 11 dogs have accepted stroking, showing no signs of aggressiveness stemming from fear. Stroking represented a positive activity for them, thus receiving a 3; 4 accepted the touch, but showed signs of anxiety, being marked with a 2 , while 5 refused this type of interaction by stepping away from the evaluator (Fig. 3).

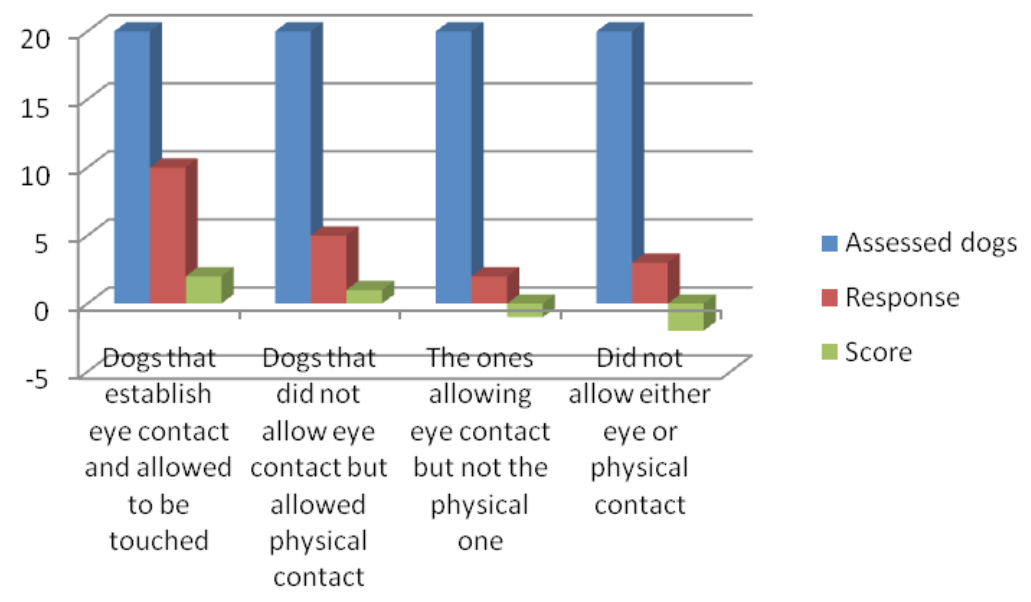

Fig. 1. Dogs response during the first stage.

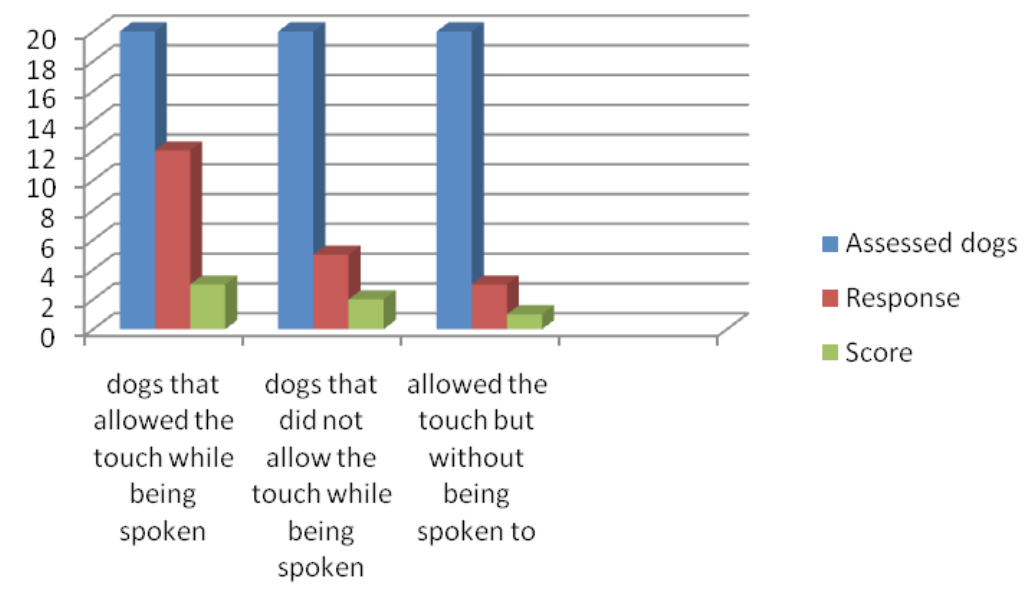

Fig. 2 Dogs response during the second stage. 


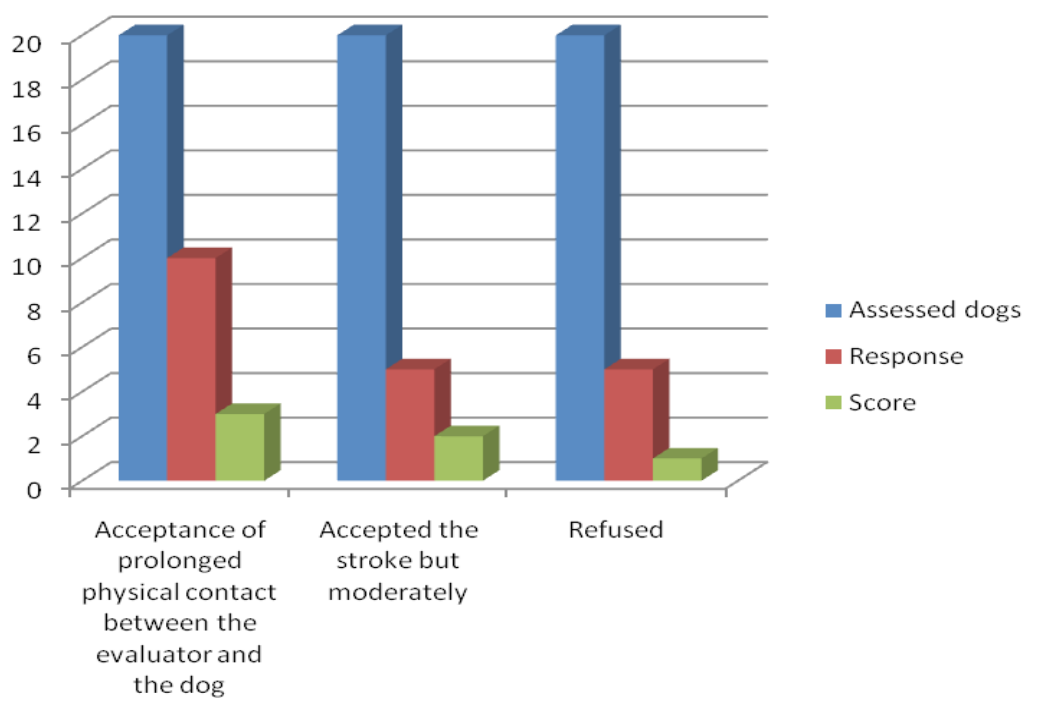

Fig. 3. Dogs response during the last stage.

\section{CONCLUSIONS}

In view of the evaluation, dogs accepting the interaction with the evaluator and not manifesting any behaviour disorders were considered apt to be put up for adoption. They did not show any muscular tension, their eyes were alert, playful, and they displayed vigorous tail wagging.

Dogs which manifested fear, anxiety for people, displaying defensive behaviour, responded by stepping away from the evaluator, turning their body in the opposite direction from they were approached from, adopting a defensive body stance: lowered head, ears flat on their head, tensed muscles around the muzzle, fearful eyes, the weight centre distributed on the back legs, drawn in tail, C body line arch, C-shaped body arch, hairs raised in the croup and tail region.

Anxious dogs did not manifest aggressiveness, but through the defensive behaviour manifested, they express obvious signs of the discomfort felt in their environment.

The dogs analyzed in the present study did not manifest aggressiveness, since the testing stopped the moment they displayed obvious anxiety and discomfort signs.

Due to the environmental conditions, shelter dogs may respond unfavourably, with defensive aggressiveness. Body language knowledge, as well as defensive dog behaviour recognition, helps avoid unpleasant situations when dogs become aggressive.
This type of aggressiveness is due to anxiety and fear, but also due to former experiences the dog has lived through. Behavioural therapy may amend behaviour disorders, but it will be difficult to offer these dogs up for adoption, since one cannot appreciate the injuriousness degree in a new environment.

Acknowledgments. This paper was published under the frame of European Social Fund, Human Resources Development Operational Programme 2007-2013, project no. POSDRU/159/1.5/ S/132765.

\section{REFERENCES}

1. Bollen K, Horowitz J (2008). Behavioral evaluation and demographic information in the assessment of aggressiveness in shelter dogs. Applied Animal Behaviour Science 112: 120-135.

2. Christensen E, Scarlett J, Campagna M, Houpt K (2007). Aggressive behavior in adopted dogs that passed a temperament test. Applied Animal Behaviour Science 106:85-95.

3. De Meester RH, De Bacquer D, Peremans K, Vermeire S, Planta DJ, Coopman F, Audenaert K (2008). A preliminary study on the use of the Socially Acceptable Behavior test as a test for shyness/ confidence in the temperament of dogs. Journal of Veterinary Behavior: Clinical Applications and Research 3:161-170.

4. Hsu Y, Sun L (2010). Factors associated with aggressive responses in pet dogs. Applied Animal Behaviour Science 123:108-123. 